\title{
Effectiveness of breast electrical impedance imaging for clinically suspicious breast lesions
}

\author{
Daglar $\mathrm{G}^{1}$, Senol $\mathrm{K}^{2}$, Yakut $\mathrm{ZI}^{3}$, Yuksek $\mathrm{YN}^{1}$, Tutuncu $\mathrm{T}^{1}$, Tez $\mathrm{M}^{1}$, Yesiltepe $\mathrm{CH}^{3}$ \\ Department of General Surgery, Breast and Endocrine Surgery, Ankara Numune Education \\ and Research Hospital, Ankara, Turkey. gul.daglar@gmail.com
}

\section{ABSTRACT}

OBJECTIVES AND BACKGROUND: This study was designed to compare the usefulness of the breast electrical conductivity measures performed in a surgical examination room against conventional breast screening modalities for identifying the symptomatic lesions of the breast tissue.

METHODS: A group of 181 patients were examined with Ultrasonography (USG), Mammography (MG), Electrical Impedance Scanning (EIS) modalities and were followed-up 24 months to clarify in terms of the lesion tumour progression relationship. Tumour biopsy was determined as an endpoint of the study.

RESULTS: According to USG, 13 (7.2 \%) lesion were suspicious, where as EIS was reported 22 (12.2 \%). 2 of these 9 patients were presented as BI-RADS 4 and histopathologic result was proven as malignant disease during 6 months short-interval follow-up. EIS exhibited compatible sensitivity (81.2\%), accuracy (84.6\%) and PPV (81.8\%) rates with USG in BI-RADS 4 subgroup, combination of these modalities raised sensitivity rates to $92.31 \%$, accuracy and PPV to $100 \%$. EIS results in BI-RADS 3 subgroup were pointed out $77.8 \%$ specificity and $87.5 \%$ NPV rates.

CONCLUSION: Breast electrical impedance measures should be useful to reduce the number of the unnecessary follow-up and biopsy rates in the clinical setting (Tab. 2, Fig. 2, Ref. 39). Text in PDF www.elis.sk.

KEY WORDS: breast, lesion, electrical impedance measures.

\section{Introduction}

Symptomatic and suspicious breast lesions are the most common complaints of the women of all ages. Surgeons' breast examination experience and clinical skills are usually efficient to detect the palpable mass. In the multidisciplinary approach, surgeons definitely refer palpable and suspicious breast lesions to a radiologist for advanced screening. Although, Mammography (MG) is surely more decisive radiological procedure, additional Ultrasonography (US) and Magnetic Resonance (MR) imaging modalities also remains the mainstay of the imaging surveillance.

Thus, The American College of Radiology developed a reporting system to stratify the findings diagnosed on the MG, US and also MR imaging modalities (1). The Breast Imaging and Reporting Data System (BI-RADS) consist of five subgroups, where a suspicious category IV or V lesions should be considered for a biopsy. BI-RADS III category, 'probably benign lesions' of the breast, have usually been undertaken for short-term screening surveillance by

${ }^{1}$ Department of General Surgery, Breast and Endocrine Surgery, Ankara Numune Education and Research Hospital, Ankara, Turkey, ${ }^{2}$ Department of General Surgery, Bursa State Hospital, Bursa, Turkey, and ${ }^{3}$ Department of Radialogy, Ankara Atatürk Research and Training Hospital, Ankara, Turkey

Address for correspondence: G. Daglar, MD, Department of General Surgery, Breast and Endocrine Surgery, Ankara Numune Education and Research Hospital, Ankara, Turkey, B Blok, zemin kat no: 32, Talatpasa Bulvarı, Sihhiye, Ankara, Turkey.

Phone: +905324336094 six month intervals within two years (2). Although, approximately $70 \%$ of patients participated the short-term screening surveillance, planning an immediate work-up schedule or screening surveillance is still a matter of debate in the clinical setting (2-6). Some authors strongly recommend that BI-RADS III lesions should be considered with USG and MG modalities together for definitive categorization, to reduce the number of short-term follow-up examinations $(7,8)$. Even though, BI-RADS III category has been associated with lower risk of malignancy, histo-pathologic sampling of these lesions presented malignant pattern ranging from $0 \%$ to $3.2 \%$ in literature (9-11). In regard with the lack of MG evaluation under 40 years old, small and non-palpable probably benign lesions should be unnoticed with either clinical and/or self breast examination and screening surveillance, until they have grown sufficiently large enough that the woman herself detects a palpable mass $(12,13)$. In addition, there are some drawbacks to perform MG for patients under 40, due to lower incidence of breast cancer and lower sensitivity as a result of an increased breast density (14). Therefore, some lesions evaluated as benign in the screening modalities, but presented with suspicious findings in the clinical examination, are elected for an immediate work-up by surgeons, who carry the same concerns with their patients $(15,16)$. Though, surgeons seek for additional accurate and feasible imaging modalities to perform during the initial contact with the patient at the examination room, which helps to raise the diagnostic sensitivity and specificity of the MG/USG and also to reduce the rates of unnecessary biopsies. There have been several imaging modalities 


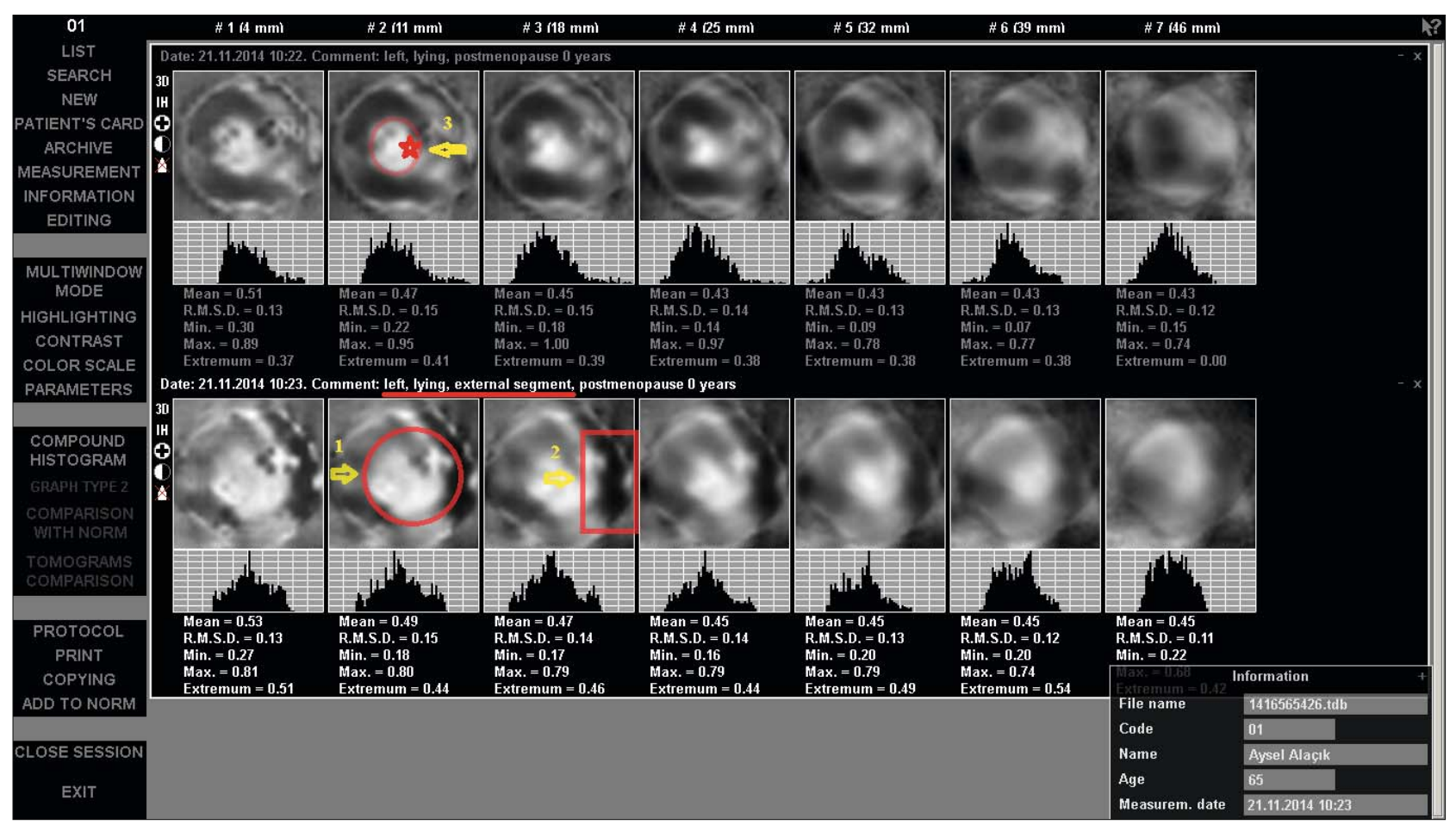

Fig. 1. Electrical impedance scanning of a suspicious BI-EIM V/ BI-RADS IV lesion.

On left breast, for the breast glands that were excluded from frontal shooting, external quadrant shooting was performed. In external quadrant shooting, at the point shown by arrow sign on row 1, column 2, sign 1, so on outer quadrant of left breast at the level of 4 o'clock, a suspected lesion of disturbed shaped, and circulated with hyperimpedance due to the compression of tissues on right part (row 2, column 2, sign 2) was observed. Its disturbed shape, being circulated with a clear hyper-impedance and on frontal shooting (row 2, column 2, sign 3), dislocation of the nipple to the left due to the lesion show that this lesion is a malign formation that is progressed; compressed and dislocated the surrounding tissues.

described for adjunctive diagnosis of the breast lesions to assist the MG and USG (17-19). Among other screening modalities routinely performed, electrical impedance scanning (EIS) is a new, non-invasive, radiation-free imaging modality (20).

The purpose of this study was to determinate the prognostic value of the breast EIS over symptomatic breast lesions classified as BI-RADS category III and IV in mammography compared to USG.

\section{Materials and methods}

\section{Diagnostic evaluation}

Mammography and ultrasonography were evaluated by experienced radiologist who was unaware of clinical findings and EIS results. EIS was performed with a 3D EIS imaging system MEIC, by a trained surgeon, who attended a training session including device technical aspects of utilization and appropriate study application. MEIC device applies an alternative current $(0.5 \mathrm{~mA})$ with a frequency of $50 \mathrm{kHz}$ to the breast tissue by scanning every $8 \mathrm{~mm}$ with the depth of $8 \mathrm{~cm}$, via annular arrayed 256 electrodes. The distributions of the electric potentials representing the breast tissue heterogeneity and conductivity rates were processed with a software program into histograms. Each histogram represents a map of hypo- and/or hyper-impedance characteristics of the breast tissue. The histograms help the clinicians to compare the scanning results of the patients for each suspicious lesion with the reference measures. The conductivity measures depending on the lesion shape, hyper-impedance margin, breast heterogeneity, local/relative electrical conductivity rates and surrounding tissue specifications were scored with between 0 to 2 values for each alteration or pathologic finding. The sum of the scores were stratified into a 5-grade scale impedance score (BI-EIM), in great concordance with BI-RADS classification system. BI-EIM score II and III were accepted as negative, where score IV and V were considered as positive and referred for a biopsy (Fig. 1).

\section{Patients}

A group of 181 patients with several breast complaints were included into a prospective cohort observational study design between January 2011 and June 2013 under an institutional review board-approved protocol. Each patient provided a written informed consent. The patient demographic characteristics, family history, clinical parameters, outcomes, imaging results and pathology results were recorded. All of the patients were evaluated with a MEIC (electrical impedance computer mammograph) device by an experienced and trained surgeon after an initial clinical breast examination. The patients provided such circumstances that have BI-EIM III and BI-RADS III lesions, were administrated for a 
short term 6-month interval imaging surveillance program. Expert radiologist evaluated the patients with the complementary use of USG, MG and MR screening modalities during 24-month period to clarify the process of the suspicious lesion progression into invasive disease. The USG was performed in all 181 patients and MG in 154 patients. Only three patients were evaluated with MR. The suspicious lesions in either clinical examination or screening modalities evaluated as BI-RADS III-V category, were sampled by true-cut or open biopsy. The EIS examinations were then compared with other imaging modalities through imaging-based and/ or pathology-based outcomes. The exclusion criteria were stated as suspicion of malignancy, pregnancy, lactation, electric-powered implants, chemotherapy, and a biopsy within 3 months of the EIS examination.

\section{Statistical analysis}

Performance characteristics of each imaging modality, including sensitivity, specificity, PPV, NPV, and accuracy, were calculated based on the histo-pathological diagnosis. We compared categorical results using a Fisher exact test and continuous variables using a 2-tailed Student $t$ test or the Wilcoxon signed rank test, as appropriate. The Chi-squared test and/or Fisher exact test was performed for a comparison of BI-RADS lexicon and the EIS results. $\mathrm{P}$ values were determined with the chi-squared test and were considered to indicate a statistically significant difference if $\mathrm{p}$ value was lower than 0.05 . Furthermore, a receiver operating curve (ROC) analysis was performed for BI-RADS and BI-EIM lexicon and a combination of both scoring systems. Data were administered and analyzed using the Statistical Package for Social Sciencers (SPSS, Release 11.5, Chicago, IL).

\section{Results}

The mean age of the patients was $43.9 \pm 10.1(18-85)$ years. The most of the breast complaints were stated as localized pain in $92(50.82 \%)$ and palpable mass in $56(30.93 \%)$ on admission. BI-
RADS III-probably benign changes and BI-RADS IV-suspicious abnormalities were identified in $166(92.26 \%)$ and in $15(8.28 \%)$ patients, respectively. BI-EIM represented benign findings in 159 (87.80 \%) and suspicious findings in $22(12.2 \%)$ patients. The ACR classification of the breast tissue was type 1 in 64 (35.4\%), type 2 in $20(11 \%)$, type 3 in $6(3.3 \%)$ and type 4 in $79(43.6 \%)$ patients. 12 (6.62 \%) BI-RADS III lesions, and 13 (7.18\%) BIRADS IV lesions were sampled. The mean lesion size was 12.6 $\pm 8.9 \mathrm{~mm}$ (benign, $19.5 \pm 13.9 \mathrm{~mm}$; malignant, $11.8 \pm 8.8 \mathrm{~mm}$ ). Benign diseases were fibroadenoma in 83 (45.8\%), hyperplasia/ metaplasia in $5(2.8 \%)$, cystic lesions in 60 (33.1\%), adenosis in $20(11 \%)$, lactation in $1(0.6 \%)$ patients. Subsequently, 14 (56 $\%$ ) invasive ductal carcinoma results were distributed as 11 (44 $\%$ ) in BI-RADS IV, and 3 (12\%) in BI-RADS III group, where 12 (48 \%) benign results were distributed as $3(12 \%)$ in BIRADS IV and $9(36 \%)$ in BIRADS III group. During 6-month short interval follow-up period, BI-EIM positive-BI-RADS negative two (1.81 $\%$ ) of nine patients were presented as BI-RADS IV and immediate work-up of these patients proved invasive malignant disease.

EIS was true positive in 12 of 14 malignancies and true negative in 7 of 11 benign lesions. Sensitivity, specificity, positive predictive value, negative predictive value and disease prevalence rates for EIS were $75 \%, 77.78 \%, 85.71 \%, 63.64 \%$ and $64 \%$, respectively. Although, pathology-based outcomes of each screening modality presented similar rates, adjunctive use of EIS with these modalities showed the highest sensitivity and PPV rates reaching $93.75 \%, 88.2 \%$, respectively $(\mathrm{p}=0.0005)$ (Tab. 1$)$.

In the BI-RADS III subgroup, the EIS was true positive in 3 of 4 malignancies and true negative in 7 of 8 benign lesions. Another noteworthy finding of EIS in BIRADS III group is that with results of one false positive and one false negative, the specificity and NPV of the screening modality reaches the rates of $87.5 \%$ (Tab. 2). The cystic lesions were better diagnosed with the USG and MG. Of 94 cystic and solid lesions, EIS were reported 1 (1.7\%) lesion as normal, $61(64.9 \%)$ lesions as cystic and solid, 32 (34\%) le-

Tab. 1. Overall 25 pathology-based outcomes of each screening modality either alone or complementary use.

\begin{tabular}{|c|c|c|c|c|c|c|c|c|c|c|}
\hline & $\begin{array}{c}\text { True } \\
\text { positive }\end{array}$ & $\begin{array}{c}\text { True } \\
\text { negative }\end{array}$ & $\begin{array}{c}\text { False } \\
\text { positive }\end{array}$ & $\begin{array}{c}\text { False } \\
\text { negative }\end{array}$ & $\begin{array}{c}\text { Sensitivity } \\
(\%)\end{array}$ & $\begin{array}{c}\text { Specificity } \\
(\%)\end{array}$ & PPV (\%) & NPV (\%) & $\begin{array}{c}\text { Disease } \\
\text { prevelance (\%) }\end{array}$ & $\mathrm{p}$ \\
\hline $\mathrm{MG}$ & 12 & 7 & 1 & 4 & 75.00 & 87.50 & 92.31 & 63.64 & 66.67 & 0.0037 \\
\hline USG & 12 & 8 & 1 & 4 & 75.00 & 88.89 & 92.31 & 66.67 & 64.00 & 0.0036 \\
\hline EIS & 12 & 7 & 2 & 4 & 75.00 & 77.78 & 85.71 & 63.64 & 64.00 & 0.0168 \\
\hline $\begin{array}{l}\text { Complementary Use of } \\
\text { EIS-USG }\end{array}$ & 15 & 7 & 2 & 1 & 93.75 & 77.78 & 88.24 & 87.50 & 64.00 & 0.0005 \\
\hline
\end{tabular}

Tab. 2. Pathology based outcomes of each screening modality, either alone or complementary use in BI-RADS III and IV group.

\begin{tabular}{|c|c|c|c|c|c|c|c|c|c|c|}
\hline & $\begin{array}{c}\text { True } \\
\text { positive }\end{array}$ & $\begin{array}{c}\text { True } \\
\text { negative }\end{array}$ & $\begin{array}{c}\text { False } \\
\text { positive }\end{array}$ & $\begin{array}{c}\text { False } \\
\text { negative }\end{array}$ & $\begin{array}{l}\text { Sensitivity } \\
(\%)\end{array}$ & $\begin{array}{l}\text { Specificity } \\
(\%)\end{array}$ & PPV (\%) & NPV (\%) & $\begin{array}{c}\text { Disease } \\
\text { prevelance }(\%)\end{array}$ & $\mathrm{p}$ \\
\hline EIS in BI-RADS 3 category & 3 & 7 & 1 & 1 & 75.00 & 87.50 & 75.00 & 87.50 & 33.33 & 0.066 \\
\hline BI-RADS 4 category & 12 & 0 & 1 & 0 & 100 & na & 92.31 & na & 92.31 & na* \\
\hline BI-EIM 4 category & 9 & 0 & 1 & 3 & 75.00 & na & 90.00 & na & 92.31 & na \\
\hline $\begin{array}{l}\text { Complementary Use in BI- } \\
\text { RADS } 4 \text { category }\end{array}$ & 12 & 0 & 1 & 0 & 100 & na & 92.31 & na & 92.31 & na \\
\hline
\end{tabular}




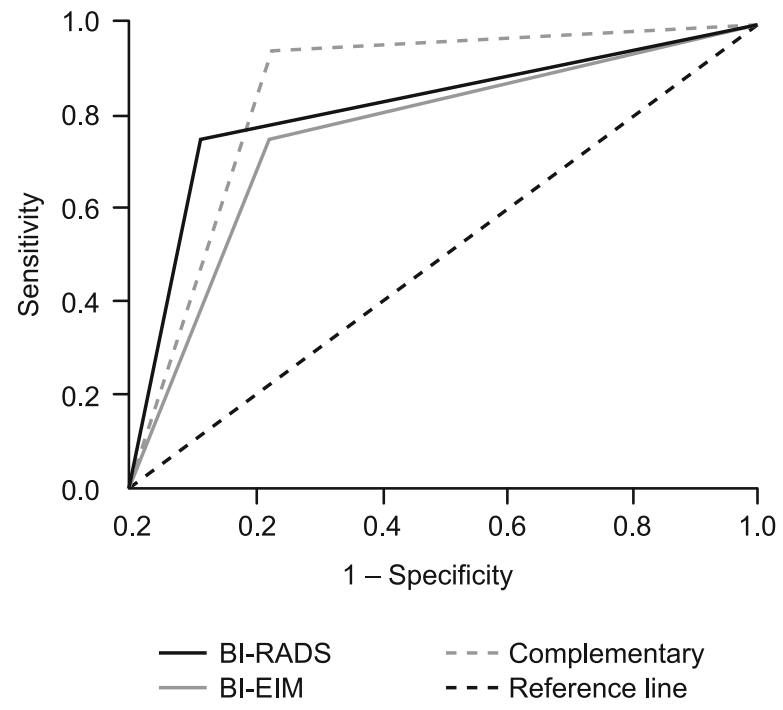

Fig. 2. Receiver operating characteristic curves of BI-RADS and BIEIM lexicon with single and complementary diagnostic performances for pathology-based outcomes.

sions as solid masses. The sensitivity, specificity, disease prevalence, positive predictive value and negative predictive value of EIS for cystic lesions were, $85.92 \%, 37.25 \%, 58.20 \%, 65.59 \%$ and $65.52 \%$, respectively. Receiver Operating Curve (ROC) analyses for diagnostic performance of MG, USG and EIM screening tests presented Area Under Curve (AUC) results of 0.819, 0.764, respectively. When the tests were administered in complementary use, the AUC reached 0.858 , with a significant $\mathrm{p}$ value $(<$ 0.004) (Fig. 2).

\section{Discussion}

EIS has been recently described in many articles as an invaluable and efficient screening modality for benign and malignant diseases of the breast (21-27). EIS evaluates the electrical activity of the breast and surrounding tissue so that lower electrical impedance-conductivity rates of the lesion compared to the surrounding tissue represent a malignant disease (28). Besides the structural findings of the lesions described through the assistance of USG and MG, evaluating the characteristics of breast tissue electrical conductivity rates have been considered as a useful data for the clinicians to guide diagnosis and treatment decisions, as well. Thus, it has been argued in literature that the EIS technique should be utilized for assessing the breast tissue as a reliable tool both in single and complementary use $(22,29,30)$.

Although EIS is emerging as a clinically useful diagnostic tool, there are brief reports evaluating the effectiveness of this screening modality (20). Among other supplementary diagnostic imaging modalities, EIS has the remarkable over-all sensitivity exceeding to $90 \%$. Malich et al. have suggested EIS with notable sensitivity rates for verification of suspicious mammographic and/ or sonographic lesions (31). However, Diebold et al have dem- onstrated overall lower sensitivity and specificity rates except for tumours smaller than $10 \mathrm{~mm}$ (32). Fuchsjaeger et al have also showed a better sensitivity and specificity rates in lesions sized $\leq 10 \mathrm{~mm}$ and invasive cancers $(30,33)$. In regard with supplementary electrical impedance of the breast, Raneta et al have reported similar outcomes with an emphasis over effectiveness of the techniques' capacity about the metabolic process, not the structural changes of the breast $(34,35)$. Therefore, breast tissue electrical conductivity rates have been favoured in suspicious lesions and EIS had been implemented into the clinical practice as an adjunctive imaging modality. In our study, adjunctive use of EIS in suspicious breast lesions had increased the sensitivity rates from $75 \%$ to $93.75 \%$ and NPV rates from $67 \%$ to $88 \%$, but presented similar results in specificity and PPV. During followup, EIS demonstrated an abnormal electrical conductivity rates in three sonographically normal cases and biopsy proved invasive cancer in two of them. Our findings indicated that EIS was a safe and accurate diagnostic tool even in unnoticed lesions by conventional imaging modalities.

There is an ongoing debate between clinicians about referring BI-RADS III category lesions to immediate work-up and/ or short term imaging follow-up $(7,36)$. Lee et al demonstrated in the study that screening surveillance of BI-RADS III category lesions was more cost-effective than the immediate surgical intervention and also spared the women from unnecessary biopsies (37). However, despite all efforts for close follow-up, the studies mentioned that approximately $30 \%$ of participants did not obey to screening surveillance (2). Helvie et al reported that 12 $\%$ patients with probably benign mammographic findings had no follow-up, and only $47 \%$ completed 3 years of surveillance (5). Similar follow-up participation rates were also reported after percutaneous biopsy of the suspicious lesions $(38,39)$. Although a large majority of BI-RADS III lesions referred for biopsy have a benign disease, some clinicians still prefer to sample the suspicious lesions. Lack of mammographic evaluation especially in younger patients states as a valid reason for biopsy requirement to detect an early breast cancer. Stojadinovic et al suggested that EIS has presented promising results for early detection of nonpalpable breast cancer in younger patient groups and described EIS as a safe diagnostic tool in this group of patients (13). The authors have also suggested the presence of the positive EIS results in biopsy proved benign lesions were significantly higher than in normal, asymptomatic women, indicating that abnormal breast impedance rates should be targeting the women at risk of a possible malignancy and should be determined as a precursor of a possible malignancy. Nonetheless, according to guidelines, the additional value of breast electrical impedance in BIRADS III lesion is not yet clear. Malich et al have demonstrated the specificity rates of EIS in sonographically visible and not visible benign lesions as $63 \%$ and $67 \%$, respectively (25). Wersebe et al have also demonstrated comparable specificity rates in proliferative and non-proliferative benign breast lesions as $67 \%$ and $71 \%$, respectively (26). These reports have demonstrated similar, but insufficient outcomes to evaluate true negative results in mammographic BIRADS III lesions 13. In contrast, recent studies have 
reported a negative breast EIS with high NPV of 83.8-97 \% as an invaluable tool to safely exclude malignancy $(30,33)$. As a result, Fuchsjaeger et al also reported that EIS should be considered as an adjunctive imaging modality in combination with USG for suspicious lesions to minimize the costs and patient morbidity. In our study, complementary use of USG and EIS reduced the false negative results of USG from $16 \%$ to $1 \%$. Our EIS results provided sufficient NPV (87.50\%) in BI-RADS III lesions to reduce the short term imaging follow-up rates by ruling out the potential malignancy risk with category minimization. Additionally high specificity and NPV rates of EIS also demonstrated that adjunctive use of these modalities should prevent unnecessary biopsy requirement for all equivocal findings.

Limitations of our study were the restriction of the analysis to BI-RADS III and IV cases and ethical problems in regard to sample all cases in BI-RADS III group. Therefore, no definitive statement about the sensitivity and specificity of breast USG could be made in BI-RADS III category lesions. Immediate work-up could not be performed with EIS results. Further analysis should evaluate suspicious lesions detected or missed by breast EIS as a first-line tool.

\section{Conclusion}

Clinicians demand additional and innovative imaging modalities through the complimentary use with the MG and USG, to reach more accurate and faster diagnostic results for the suspicious breast lesions. In regards to the new advances with more comprehensive works, the EIS modality would be a standard tool (first step tool) for the management of the breast lesions in the near future.

\section{References}

1. Mendelson E, Baum J, Berg W, Merritt C, Rubin E. Breast imaging reporting and data system, BI-RADS: ultrasound. Reston, VA: American College of Radiology, 2003.

2. Baum JK, Hanna LG, Acharyya S et al. Use of BI-RADS 3-probably benign category in the American College of Radiology Imaging Network Digital Mammographic Imaging Screening Trial. Radiology 2011; 260: 61-67.

3. Kapsimalakou S, Waldmann A, Katalinic A et al. Follow-up of probably benign lesions in non-screening breast diagnostics. Arch Gynecol Obstetrics 2014.

4. Pisano ED, Yankaskas BC, Ghate SV, Plankey MW, Morgan JT. Patient compliance in mobile screening mammography. Acad Radiol 1995; 2: $1067-1072$.

5. Helvie MA, Pennes DR, Rebner M, Adler DD. Mammographic follow-up of low-suspicion lesions: compliance rate and diagnostic yield. Radiology 1991; 178: 155-158.

6. Yabroff KR, Breen N, Vernon SW, Meissner HI, Freedman AN, Ballard-Barbash R. What factors are associated with diagnostic followup after abnormal mammograms? Findings from a U.S. National Survey. Cancer epidemiology, biomarkers \& prevention: a publication of the American Association for Cancer Research, cosponsored by the American Society of Preventive Oncology 2004; 13: 723-732.
7. Sickles EA. Probably benign breast lesions: when should follow-up be recommended and what is the optimal follow-up protocol? Radiology 1999; 213: 11-14.

8. Varas X, Leborgne JH, Leborgne F, Mezzera J, Jaumandreu S, Leborgne F. Revisiting the mammographic follow-up of BI-RADS category 3 lesions. AJR. Amer J Roentgenol 2002; 179: 691-695.

9. D’Orsi CJ. To follow or not to follow, that is the question. Radiology 1992; 184: 306.

10. Rubin E. Six-month follow-up: an alternative view. Radiology 1999; 213: $15-18$.

11. Hall FM. Malignancy in BI-RADS category 3 mammographic lesions. Radiology 2002; 225: 918-919.

12. Park BW, Kim SI, Kim MH, Kim EK, Park SH, Lee KS. Clinical breast examination for screening of asymptomatic women: the importance of clinical breast examination for breast cancer detection. Yonsei Med J 2000; 41: 312-318.

13. Stojadinovic A, Nissan A, Shriver CD et al. Electrical impedance scanning as a new breast cancer risk stratification tool for young women. J Surg Oncol 2008; 97: 112-120.

14. Barlow WE, Lehman CD, Zheng Y et al. Performance of diagnostic mammography for women with signs or symptoms of breast cancer. $\mathrm{J}$ National Cancer Inst 2002; 94: 1151-1159.

15. Orel SG, Kay N, Reynolds C, Sullivan DC. BI-RADS categorization as a predictor of malignancy. Radiology 1999; 211: 845-850.

16. Mendez A, Cabanillas F, Echenique M, Malekshamran K, Perez I, Ramos E. Mammographic features and correlation with biopsy findings using 11-gauge stereotactic vacuum-assisted breast biopsy (SVABB). Ann Oncol 2004; 15: 450-454.

17. Xydeas T, Siegmann K, Sinkus R, Krainick-Strobel U, Miller S, Claussen CD. Magnetic resonance elastography of the breast: correlation of signal intensity data with viscoelastic properties. Invest Radiol 2005; 40: 412-420.

18. Joe BN, Chen VY, Salibi N, Fuangtharntip P, Hildebolt CF, Bae KT. Evaluation of 1H-magnetic resonance spectroscopy of breast cancer pre- and postgadolinium administration. Invest Radiol 2005; 40: 405-411.

19. Floery D, Helbich TH, Riedl CC et al. Characterization of benign and malignant breast lesions with computed tomography laser mammography (CTLM): initial experience. Invest Radiol 2005; 40: 328-335.

20. Vreugdenburg TD, Willis CD, Mundy L, Hiller JE. A systematic review of elastography, electrical impedance scanning, and digital infrared thermography for breast cancer screening and diagnosis. Breast Cancer Res Treat 2013; 137: 665-676.

21. Martin G, Martin R, Brieva MJ, Santamaria L. Electrical impedance scanning in breast cancer imaging: correlation with mammographic and histologic diagnosis. Eur Radiol 2002; 12: 1471-1478.

22. Stojadinovic A, Nissan A, Gallimidi Z et al. Electrical impedance scanning for the early detection of breast cancer in young women: preliminary results of a multicenter prospective clinical trial. J Clin Oncol 2005; 23: 2703-2715.

23. Cherepenin V, Karpov A, Korjenevsky A et al. A 3D electrical impedance tomography (EIT) system for breast cancer detection. Physiol Meas 2001; 22: 9-18.

24. Cherepenin VA, Karpov AY, Korjenevsky AV et al. Three-dimensional EIT imaging of breast tissues: system design and clinical testing. IEEE Trans Med Imaging 2002; 21: 662-667. 


\section{5-510}

25. Malich A, Fuchsjager M. Electrical impedance scanning in classifying suspicious breast lesions. Invest Radiol 2003; 38: 302-303.

26. Wersebe A, Siegmann K, Krainick U et al. Diagnostic potential of targeted electrical impedance scanning in classifying suspicious breast lesions. Invest Radiol 2002; 37: 65-72.

27. Mammography and Beyond. Developing Technologies for the Early Detection of Breast Cancer: The National Academies Press; 2001.

28. Malich A, Boehm T, Facius M et al. Differentiation of mammographically suspicious lesions: evaluation of breast ultrasound, MRI mammography and electrical impedance scanning as adjunctive technologies in breast cancer detection. Clin Radiol 2001; 56: 278-283.

29. Prasad SN, Houserkova D, Campbell J. Breast imaging using 3D electrical impedence tomography. Biomedical papers of the Medical Faculty of the University Palacky, Olomouc, Czechoslov 2008; 152: 151-154.

30. Fuchsjager MH, Helbich TH, Ringl $\mathbf{H}$ et al. Electrical impedance scanning in the differentiation of suspicious breast lesions: comparison with mammography, ultrasound and histopathology. Rofo 2002; 174: 1522-1529.

31. Malich A, Fritsch T, Anderson R et al. Electrical impedance scanning for classifying suspicious breast lesions: first results. Eur Radiol 2000; 10: 1555-1561.

32. Diebold T, Jacobi V, Scholz B et al. Value of electrical impedance scanning (EIS) in the evaluation of BI-RADS III/IV/V-lesions. Technol Cancer Res Treat 2005; 4: 93-97.
33. Fuchsjaeger MH, Flory D, Reiner CS, Rudas M, Riedl CC, Helbich TH. The negative predictive value of electrical impedance scanning in BI-RADS category IV breast lesions. Invest Radiol 2005; 40: $478-485$.

34. Raneta O, Ondrus D, Bella V. Utilisation of electrical impedance tomography in breast cancer diagnosis. Klin Onkol 2012; 25: 36-41.

35. Raneta O, Bella V, Bellova L, Zamecnikova E. The use of electrical impedance tomography to the differential diagnosis of pathological mammographic/sonographic findings. Neoplasma 2013; 60: 647-654.

36. Varas X, Leborgne F, Leborgne JH. Nonpalpable, probably benign lesions: role of follow-up mammography. Radiology 1992; 184: 409-414.

37. Lee CI, Wells CJ, Bassett LW. Cost minimization analysis of ultrasound-guided diagnostic evaluation of probably benign breast lesions. Breast J 2013; 19: 41-48.

38. Pal S, Ikeda DM, Birdwell RL. Compliance with recommended follow-up after fine-needle aspiration biopsy of nonpalpable breast lesions: a retrospective study. Radiology 1996; 201: 71-74.

39. Goodman KA, Birdwell RL, Ikeda DM. Compliance with recommended follow-up after percutaneous breast core biopsy. Amer J Roentgenol 1998; 170: 89-92.

Received March 2, 2016. Accepted March 22, 2016. 